\title{
Identification of critical parameters affecting voltage and angular stability considering load-renewable generation correlations
}

DOI:

10.1109/TPWRS.2019.2891840

\section{Document Version}

Accepted author manuscript

Link to publication record in Manchester Research Explorer

Citation for published version (APA):

Qi, B., Hasan, K., \& Milanovic, J. V. (2019). Identification of critical parameters affecting voltage and angular stability considering load-renewable generation correlations. IEEE Transactions on Power Systems. https://doi.org/10.1109/TPWRS.2019.2891840

\section{Published in:}

IEEE Transactions on Power Systems

\section{Citing this paper}

Please note that where the full-text provided on Manchester Research Explorer is the Author Accepted Manuscript or Proof version this may differ from the final Published version. If citing, it is advised that you check and use the publisher's definitive version.

\section{General rights}

Copyright and moral rights for the publications made accessible in the Research Explorer are retained by the authors and/or other copyright owners and it is a condition of accessing publications that users recognise and abide by the legal requirements associated with these rights.

\section{Takedown policy}

If you believe that this document breaches copyright please refer to the University of Manchester's Takedown Procedures [http://man.ac.uk/04Y6Bo] or contact uml.scholarlycommunications@manchester.ac.uk providing relevant details, so we can investigate your claim.

\section{OPEN ACCESS}




\title{
Identification of Critical Parameters Affecting Voltage and Angular Stability Considering Load-Renewable Generation Correlations
}

\author{
Buyang Qi, Student Member, IEEE, Kazi N. Hasan, Member, IEEE, and Jovica V. Milanović, Fellow, \\ IEEE
}

\begin{abstract}
The renewable energy source based generating technologies and flexible demand and storage devices exhibit significant temporal and spatial uncertainties in generating and loading profiles and introduce additional level of uncertainty in network operation. The dynamic behaviours of such a network can be affected and the stable operation may be compromised. This paper proposes a probabilistic analysis approach for the evaluation of the effect of uncertain parameters on power system voltage and angular stability. Load margin, the damping of critical eigenvalues and the transient stability index (TSI) have been chosen as the relevant stability indices for voltage stability, small-disturbance stability and transient stability analysis, respectively. The Morris screening sensitivity analysis method coupled with a multivariate Gaussian copula to account for parameter correlations is used for the priority ranking of uncertain parameters. The approach is illustrated on a number of case studies using modified IEEE 68-bus NETS-NYPS test system. The results obtained in this paper reveal that the critical parameters appear as groups if the input dataset is correlated, and hence even a parameter (which may be uninfluential individually) can have a significant impact on system dynamic behaviour due to its correlation with other influential parameters.
\end{abstract}

Index Terms - Copula method, power system computation, renewable generation, sensitivity analysis, small-disturbance stability, transient stability, voltage stability

\section{INTRODUCTION}

$\mathrm{T}$ HE planning and operation of modern power systems have changed significantly compared to conventional power systems due to the addition of renewable energy sources (RES). The key characteristic of RES and new types of load is that their operation is highly temporal and spatial dependent. Hence additional uncertainties are introduced from both sides, the network generation and loading [1]. The variability exhibited by these new technologies voids traditional deterministic stability analysis since the 'worst case scenario' analysis of the network may lead to an overly conservative system design [2-4]. A probabilistic approach to network stability assessment

The authors are with the School of Electrical and Electronic Engineering, The University of Manchester, PO Box 88, Manchester M60 1QD, UK. (Email: buyang.qi@manchester.ac.uk, kazi.hasan@manchester.ac.uk, jovica.milanovic @ manchester.ac.uk) is getting steadily adopted by researchers to be applied to all types of stability studies [1, 5-12]. The Monte Carlo (MC) simulations are commonly used to perform these probabilistic studies [5, 13-16].

Due to the size of the power systems and the increase in the number of uncertain parameters, high computation resources are required when MC simulation is applied to probabilistic power system stability analysis. In such cases, priority ranking of system uncertain parameters, based on their influence on different stability aspects, can help the system operators to ensure optimal dynamic performance of the system with minimal use of human and computational resources.

Previous studies employed sensitivity analysis (SA) techniques for the identification of influential parameters affecting voltage/small-disturbance stability within a network with RES generation [17-19]. The influence of load models, uncertainty in loading demand and RES generations on power system stability is analyzed in these studies. The advanced Morris screening sensitivity analysis method (MSSA) is compared with the commonly used local sensitivity analysis method (LSA) and the global sensitivity analysis method (GSA) and its efficiency and accuracy have been demonstrated. This paper builds on those studies and expands the application of MSSA to the priority ranking of critical system parameters affecting the transient stability of the network with RES generation in addition to voltage and small-disturbance stability.

The research discussed above employs independent probability distributions for the modelling of uncertain parameters. The random sampled data set obtained in this way however, does not represent the correlations among uncertainties within the real system, hence the results of the analysis may not be accurate enough [20-26]. The modelling of correlations among system parameters can be done effectively using Copula theory [26]. Different dependence structures among the correlated parameters can be modelled by using different copula families. The performance of six different copula approaches, (i) Gaussian (ii) Student (iii) Clayton (iv) Frank (v) Gumbel and (vi) multivariate joint normal distribution, was compared in [26] and the application of different copulas to different dimensional systems in [27]. It was reported in [26] that the multivariate Gaussian copula is the most effective and accurate for representation of the correlations between uncertainties involved in modelling load demand, wind generation and PV generation for power system small-disturbance stability analysis. In [28], the Gaussian 


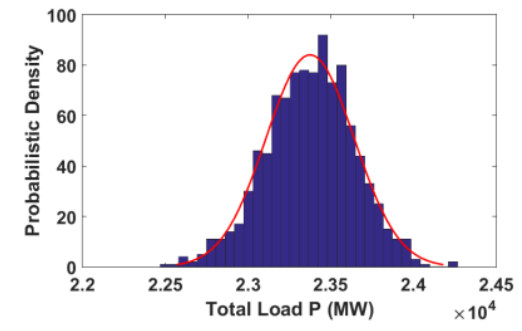

Fig. 1. Histogram-pdf of nose-point locus for voltage stability analysis based on 1000 Monte Carlo simulations

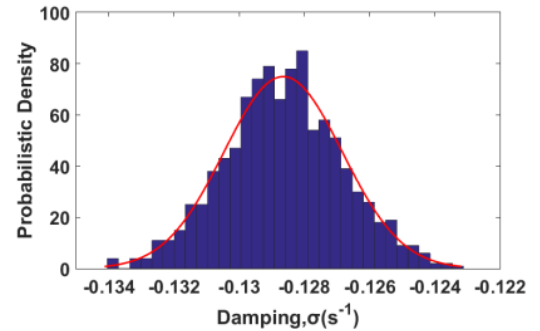

Fig. 2. Histogram- $p d f$ of the damping of the critical mode for small-disturbance stability analysis based on 1000 Monte Carlo simulations

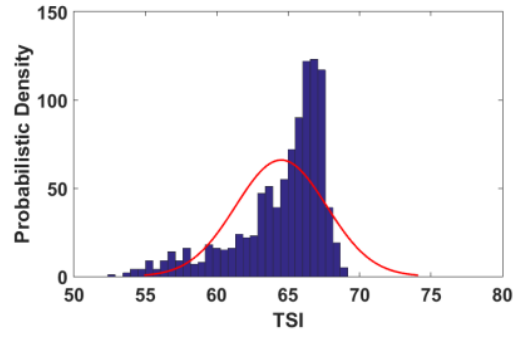

Fig. 3. Histogram- $p d f$ of TSI for transient stability analysis based on 1000 Monte Carlo simulations copula has been employed for voltage stability analysis. The multivariate Gaussian copula is applied here first to uncertainty modelling for transient stability analysis and then for voltage and small disturbance stability analysis.

This paper uses the load margin, damping of the critical eigenvalues, and transient stability index (TSI) as stability indices for voltage, small-disturbance and transient stability analysis, respectively. The uncertainties of RES generation and loading (following the daily loading curve) are modelled probabilistically. The Morris screening sensitivity analysis (MSSA) method is applied for the priority ranking of critical parameters, while the copula approach is used to model the correlation between input parameters. The paper clearly demonstrates that i) the critical system parameters affecting voltage and angular stability in power systems with renewable generation can be identified through MSSA; ii) the correlation between input parameters can be effectively modelled using a multivariate Gaussian copula approach; and iii) it is important to consider correlation between uncertain parameters for system voltage and angular stability assessment.

This paper for the first time combines and validates the identification of critical parameters affecting system stability globally, i.e., voltage stability and small and large disturbance angular stability simultaneously, by using efficient sensitivity analysis method, Morris Screening sensitivity analysis method. It also for the first time establishes the importance of modelling parameter correlation by using Copula theory for identification of critical system parameters affecting global system stability. The approach facilitates efficient identification of important system parameters that need to be accurately modelled for reliable system stability studies and such ensures cost effective use of human and financial resources by system operators.

\section{Voltage AND ANGUlar Stability}

Voltage stability problems are commonly found in heavily loaded networks as the reactive power provided by the system may not be sufficient to support the user-end voltages [29]. A commonly used approach for the assessment of power system voltage stability is the PV-curve analysis, where the stability index is the loadability (or load margin) of the system [7, 17, 28]. This index indicates the maximum active power the network can provide to meet the load demand before the voltage collapse. Fig. 1 shows the histogram of critical loadability values obtained from $1000 \mathrm{MC}$ simulations and the fitted probability density function $(p d f)$, corresponding to normal distribution in this case, to illustrate the variation in system loadability due to uncertainties in load and RES.
For power system small-disturbance stability analysis, the damping of the critical oscillatory mode is used as the stability index, as defined in (1) [14].

$$
\xi_{i}=\frac{-\sigma_{i}}{\sqrt{\sigma_{i}^{2}+\omega_{i}^{2}}}
$$

In (1) $\xi_{i}, \sigma_{i}$ and $\omega_{i}$ are the damping ratio, damping and frequency of the critical eigenvalue. Fig. 2 shows the histogram of the damping of the critical electromechanical mode obtained from $1000 \mathrm{MC}$ simulations and corresponding, fitted $p d f$ based on normal distribution to illustrate the effect of uncertainties on small disturbance stability.

Finally, power system transient stability is most frequently analyzed using the transient stability index (TSI) given by (2) [30-32].

$$
T S I=100 * \frac{360-\delta_{\max }}{360+\delta_{\max }}
$$

In (2) $\delta_{\max }$ is the maximum rotor angle separation between any two generators in the network after a fault. A negative TSI value indicates that the system is unstable. The larger the TSI the more stable the system is. Fig. 3 presents the histogram of the TSI obtained from $1000 \mathrm{MC}$ simulations and corresponding fitted $p d f$ to illustrate the effect of considered uncertainties on system transient stability. The TSI histogram is fitted with normal distribution as before for the ease of comparison though normal distribution clearly is not the most appropriate fit in this case.

The Figs 1-3 are given for illustrative purposes only to illustrate the results of probabilistic stability studies. They are obtained by performing 1000 corresponding MC simulations for each of the three stability studies and for a specific operating condition using the IEEE 68-bus test network described in Section IV of this paper. Different operating conditions (loading level, uncertainty level, topology, generation dispatch, etc.) would result in different shapes and spread of obtained histograms and corresponding fitted $p d f$ s. Based on the fitted $p d f$ s stability profiles of the test network can be analysed and when the stability limits are taken into consideration the risk of system instability can be assessed.

\section{Methodology}

The priority ranking of system uncertain parameters based on their influence on power system stability consists of four major steps: (i) using a proper probability distribution function $(p d f)$ in order to generate the dataset of system uncertain parameters, (ii) solving Optimal Power Flow (OPF) to determine the dispatch of conventional generation, (iii) calculating the corresponding stability index for different categories of stability problems, (iv) using the sensitivity 
analysis (SA) method to assess the system uncertainties for the ranking. Fig. 4 is a flow chart of the proposed methodology.

\section{A. Probabilistic Modelling of Uncertain Input Parameters}

In order to represent the operational conditions in a network with RES generation and uncertain loading demands, this paper employs the probabilistic modelling of system loads, wind speed and solar irradiation as uncertain parameters. Table I illustrates the probability distribution for selected system uncertainties and their corresponding model parameters. Past studies indicated that normal distribution is commonly used for modelling load uncertainty $[15,33,34]$, hence adopted in this study. Similarly, uncertainties caused by variation in wind speed and solar irradiation for wind farm and PV plants were commonly modelled with Weibull [5, 6, 35] and Beta distribution [36, 37], respectively. The normal distribution is typically represented by mean $(\mu)$ and standard deviation $(\sigma)$, Weibull distribution by scale parameter $(\alpha)$ and shape parameter $(\beta)$, and beta distribution by shape parameters (a) and (b). The level of uncertainty of $10 \%$ adopted for modelling load uncertainty is the typical load forecast error. For Weibull and beta distribution the parameters follow a similar level of uncertainty as that for the load [15].

\section{B. Priority Ranking of System Uncertain Parameters}

The priority ranking of system uncertain parameters is obtained through performing a sensitivity analysis (SA). SA methods can numerically describe how the input uncertainties can affect the output variabilities after they go through a modelled system. Generally speaking, the SA method consists of two categories, the local sensitivity analysis method (LSA) and the global sensitivity method (GSA). Previous studies [17, 19] provide a thorough comparison between different categories of SA methods. In total 6 widely used SA methods, including One-at-A-Time (LSA), Morris Screening (GSA), Pearson correlation coefficient (GSA), Spearman correlation coefficient (GSA), Partial correlation coefficient (GSA), and Sobal total indices (GSA), are applied in the identification of critical parameters for voltage and small-disturbance stability analysis. Both studies recommended the Morris screening method as the most suitable for this purpose. It performs designated semi-global search among input variabilities as an efficient approach for the priority ranking of uncertain parameters.

The sensitivity indices for the MSSA method are the mean $\left(\mu^{*}\right)$ and the standard deviation $\left(\sigma^{*}\right)$ of the elementary effects $\left(E E_{p}^{i}\right)$ of individual input uncertainty, as defined in (3), (4) and (5) [38].

$$
\begin{gathered}
\mu_{p}^{*}=\frac{1}{r} \sum_{i=1}^{r}\left|E E_{p}^{i}\right| \\
\sigma_{p}^{*}=\sqrt{\frac{1}{r} \sum_{i=1}^{r}\left(\left|E E_{p}^{i}\right|-\mu_{p}^{*}\right)^{2}} \\
E E_{p}^{i}(x)=\frac{\left[y\left(x_{1}, x_{2}, \ldots, x_{i-1}, x_{i}+\Delta, x_{i+1}, \ldots, x_{p}\right)-y(x)\right]}{\Delta}
\end{gathered}
$$

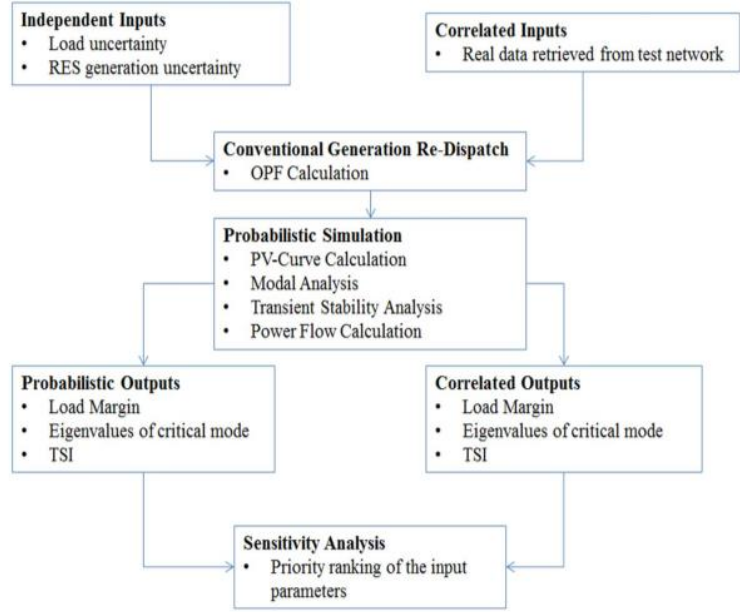

Fig. 4 Flow chart of the proposed methodology

TABLE I

PROBABILISTIC DisTRIBUTION AND MODEL PARAMETERS OF SYSTEM UNCERTAINTIES OF THE TEST NETWORK

\begin{tabular}{cccc}
\hline \hline $\begin{array}{c}\text { Uncertain } \\
\text { Parameter }\end{array}$ & $\begin{array}{c}\text { Probability } \\
\text { Distribution }\end{array}$ & $\begin{array}{c}\text { Probabilistic Model } \\
\text { Parameters }\end{array}$ & $\begin{array}{c}\text { Level of } \\
\text { Uncertainty }\end{array}$ \\
\hline $\begin{array}{c}\text { Load Demand } \\
(\%)\end{array}$ & Normal & $3 \sigma=10 \%$ of $\mu$ & \\
\cline { 1 - 2 } $\begin{array}{c}\text { Wind Speed } \\
\left(\mathrm{ms}^{-1}\right)\end{array}$ & Weibull & $\alpha=2.2, \beta=11.1$ & High (10\%) \\
\cline { 1 - 2 } $\begin{array}{c}\text { Solar Irradiation } \\
\left(\mathrm{kWm}^{-2}\right)\end{array}$ & Beta & $\mathrm{a}=13.7, \mathrm{~b}=1.3$ & \\
\hline \hline
\end{tabular}

In (3) $\mu_{p}^{*}$ serves as the ranking score for individual input uncertainties. The higher the score is, the more influential the corresponding uncertainty is considered. A high value of $\sigma_{p}^{*}$ indicates the corresponding input has a non-linear effect on the output. $p$ is the number of input uncertainties, $r$ is the 'level' of MSSA (between 4 to 10 ), and $\Delta$ is the step size determined through $\Delta=\frac{1}{r-1}$. The MSSA method requires $n=p * r+1$ simulations. The computational burden is significantly lower compared to the conventional GSA method like the Sobol total indices method [17, 19].

\section{Correlations between Input Uncertainties}

In order to consider the stochastic dependence structures between the modelled uncertainties in power systems, the copula methods can be applied to generate a correlated input dataset. In [26], six correlation modelling techniques have been compared in terms of their accuracy and efficiency. It has been demonstrated that the multivariate Gaussian (mvG) copula is the most accurate and efficient. The mvG copula has also been successfully applied in the assessment of system frequency stability, where it showed the best performance in terms of accuracy and efficiency [39]. The mvG copula is therefore selected for correlation modelling in this study. The comparison of different copula techniques is beyond the scope of this paper and it can be found in [26, 39].

Copula theory provides an effective way of modeling stochastic dependence (or correlation) between random variables. According to Sklar's theorem: any multivariate joint distribution can be written in terms of univariate marginal distribution functions and a copula which describes the dependence structure between the variables [40]. 
The copula function $C$ can be represented by the multivariate $c d f F$ (cumulative distribution function) and marginal $c d f F_{i}$, as in (6) [41].

$$
C\left[F_{1}\left(x_{1}\right), F_{2}\left(x_{2}\right), \ldots, F_{n}\left(x_{n}\right)\right]=F\left(x_{1}, x_{2}, \ldots, x_{n}\right)
$$

1) Multivariate Gaussian Copula

The multivariate Gaussian ( $\mathrm{mvG}$ ) copula is known as an elliptical copula, which provides the flexibility to model a higher number of dimensions. The mvG is very useful to model dependent random variables, when there are complicated relationships among the variables, or when the individual variables are from different distributions. As power system load, wind and solar data follow different probability distributions, the mvG has been found to be very efficient in modelling their interdependences [26, 39].

The copula function for the $\mathrm{mvG}$ copula can be expressed as in (7) [27].

$$
C\left(u_{1}, u_{2}, \ldots, u_{n} ; \Sigma\right)=\phi_{\Sigma}\left(\phi^{-1}\left(u_{1}\right), \phi^{-1}\left(u_{2}\right), \ldots, \phi^{-1}\left(u_{n}\right)\right)(7)
$$

In (7), $\Sigma$ denotes a symmetric, positive definite matrix with $\operatorname{diag}(\Sigma)=1, \Phi_{\Sigma}$ is the standard multivariate normal distribution with correlation matrix $\Sigma$, and $\Phi^{-1}(\bullet)$ is the inverse of the Normal $c d f$.

The correlation matrix $\Sigma$ in (7), also known as the covariance matrix, can be expressed as (8).

$$
\Sigma=\left[\begin{array}{cccc}
\rho_{11} & \rho_{12} & \ldots & \rho_{1 n} \\
\rho_{21} & \rho_{22} & \cdots & \rho_{2 n} \\
& \vdots & \ddots & \vdots \\
\rho_{n 1} & \rho_{n 2} & \cdots & \rho_{n n}
\end{array}\right]
$$

In (8), $\rho$ is the linear correlation coefficient between parameters to represent the range of different dependence structure. The correlation between the same set of data is given by (9).

Then eq. (8) becomes (10).

$$
\rho_{11}=\rho_{22} \ldots=\rho_{n n}=1
$$

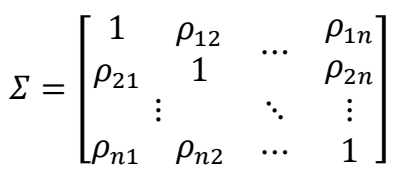

The covariance matrix can be formed with the Pearson correlation coefficient $\rho$, Spearman or Kendall rank correlation coefficient $\tau$ [42].

The inverse of the normal $c d f \Phi^{-1}(\bullet)$, as presented in eq. (7), has been adopted from the theory of univariate random number generation. The inversion method has been used here to model the individual variables, which follow different distributions. In this study, for example, system load, wind and solar data follow normal, Weibull and beta distributions, respectively. The step-by-step procedure for generating correlated random samples has been discussed in [26].

\section{D.Monte-Carlo Stopping Rule}

The MC simulation requires a certain number of repeated random sampling of uncertain data in order to represent the stochastic behaviour of system parameters. The following equation (11) determines the required Monte Carlo run [43].

$$
\varepsilon>\left[\frac{\left\{\phi^{-1}\left(1-\frac{\delta}{2}\right) \cdot \sqrt{\frac{\sigma^{2}(X)}{N}}\right\}}{\bar{X}}\right]
$$

In (11), $\varepsilon$ is the sample mean error, $\phi^{-1}(\cdot)$ is the inverse Gaussian conditional probability distribution with a zero mean value and a one standard deviation value, $\sigma^{2}(\cdot)$ is the variance of a sample, $\delta$ is the required confidence level, and $\bar{X}$ is the mean of the samples.

Previous studies on the IEEE 68-Bus NETS-NYPS test system indicated that for transient stability studies the sampling number required for a 5\% sample mean error with $99 \%$ confidence interval is 6000 simulations [31]. Also it was found that 1000 simulations are required for small disturbance stability studies with a $0.3 \%$ sample mean error and $99 \%$ confidence interval [26]. For voltage stability, 1000 simulations are required for less than $2 \%$ sample mean error and $99 \%$ confidence interval. Therefore, 6000 simulations are run in this study to ensure the required accuracy.

\section{Test Network AND Simulation}

The simulation is performed with two major software platforms, Matlab R2015a and DIgSILENT PowerFactory 2017 SP1 (x64). The probabilistic modelling of input uncertainties and the calculation of the probabilistic load flow are performed through the OPF solver within MATPOWER, Matlab [44]. The PV-curve calculation, modal analysis, and RMS simulation for stability analysis are performed in DIgSILENT PowerFactory. The obtained outputs of different stability studies are processed through the Matlab software.

\section{A. Test System Configuration}

The test network used in this paper is a modified version of the IEEE 68-Bus NETS-NYPS (New England Test System-New York Power System) [45]. There are 16 generators and 68 buses in the network. The test network is divided into 5 sections with Generator G1-G9 located in NETS and Generators G10-G13 located in NYPS. G14, G15, and G16 separate as 3 equivalent areas connected to NYPS. The test network is rated to provide an active power generation capacity of $17.26 \mathrm{GW}$ (1p.u.). Integration of renewable generation is

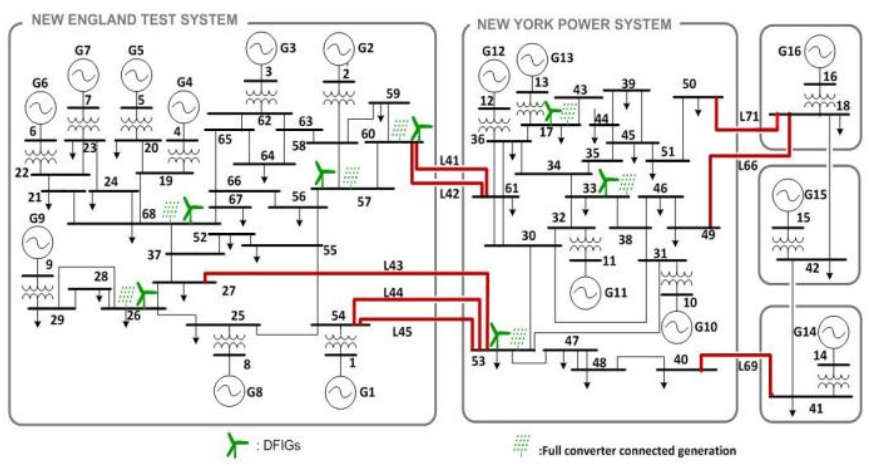

Fig. 5. Modified IEEE 68-Bus NETS-NYPS test system with RES generations.

TABLE II

THE RES GENERATION PENETRATION LEVEL UNDER VARIABLE LOADING DEMAND SELECTED FROM DAILY LOADING CURVE

\begin{tabular}{cllllllll}
\hline \hline Loading & 1.0 & 0.9 & 0.8 & 0.7 & 0.6 & 0.5 & 0.4 & 0.3 \\
Demand & p.u. & p.u. & p.u. & p.u. & p.u. & p.u. & p.u. & p.u. \\
\hline$P L_{R E S}$ & $20 \%$ & $22 \%$ & $25 \%$ & $29 \%$ & $33 \%$ & $40 \%$ & $50 \%$ & $67 \%$ \\
\hline \hline
\end{tabular}


simulated by using 7 equivalent wind generators and 7 equivalent PV generators, which are connected to 7 system buses (buses 60, 57, 68, 26 of NETS, and buses 53, 33, 17 of NYPS). More detailed data of the test network including parameter modelling can be found in [45].

Fig. 5 shows the layout of the test network. The wind turbines are modelled as Doubly Fed Induction Generators (DFIGs) and the PV-plants are modelled as Full Converter Connected Generators (FCCs). The Grid Code of National Grid requires that generators should be able to operate between a 0.85 lagging power factor and a 0.95 leading power factor under rated active power generation conditions [46]. Hence the reactive power generation limit of the synchronous generators within the test network is set between $-33 \%$ and $62 \%$ of the active power generation.

There are 68 system buses in the test network, among which 16 buses (Bus1 to Bus16) are connected to G1-G16. The variabilities in load demand $P_{i}$ (in p.u.) are modelled probabilistically on the remaining 52 buses. The total number of uncertain parameters modelled within the test network is 66 (including 14 RES generators). The total simulation run with MSSA applied is calculated as $n=p \times r+1=661$ (66 uncertainties, 10 levels). However as there are 17 buses which do not have a load connected (no uncertainty), hence the number of displayed system uncertainties being ranked using the SA method is 49 .

\section{B. Load Model and System Load Profile}

All loads in the system are modelled using classical exponential load model given by (12) and (13) [47].

$$
\begin{aligned}
& P_{L}=P_{n}\left(\frac{U}{U_{n}}\right)^{0} \\
& Q_{L}=Q_{n}\left(\frac{U}{U_{n}}\right)^{2}
\end{aligned}
$$

In (12) and (13), $P_{L}$ and $Q_{L}$ are the active and reactive power drawn by the system load at voltage $U, P_{n}$ and $Q_{n}$ are the active and reactive power drawn by the system load under rated voltage $U_{n}$.

In order to assess system behaviour under different loading conditions, 8 different loading levels have been selected along the daily loading curve varying from 1p.u. to 0.3 p.u. Equations (14) and (15) illustrate the relation between the system loading $P_{L}$, the active power of synchronous machine $G_{S M}$, and the active power of RES generation $G_{R E S}$. $P L_{R E S}$ is the penetration level of RES generation. Table II lists the operational conditions following the daily loading curve and their corresponding $P L_{R E S}$.

$$
\begin{gathered}
P_{L}=G_{S M}+G_{R E S} \\
P L_{R E S}=\frac{G_{R E S}}{P_{L}}
\end{gathered}
$$

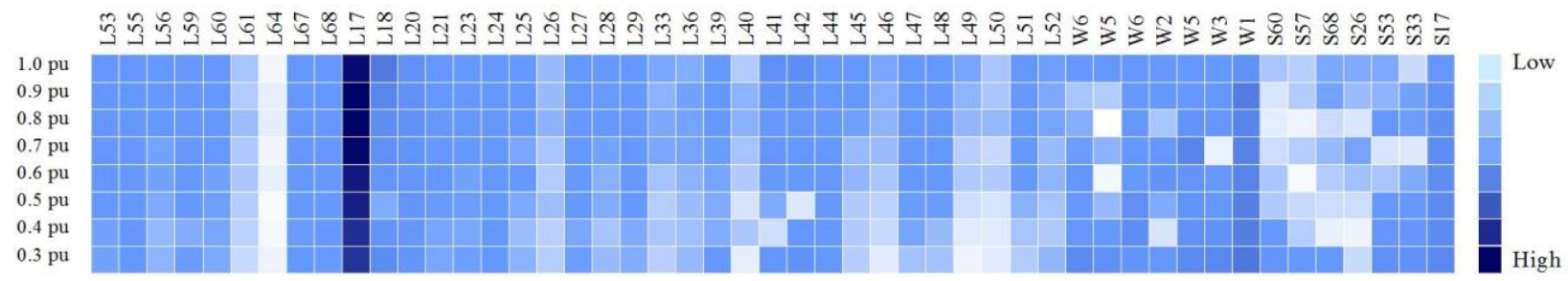

Fig. 6. Heatmap of ranking for voltage stability index showing different system loading.

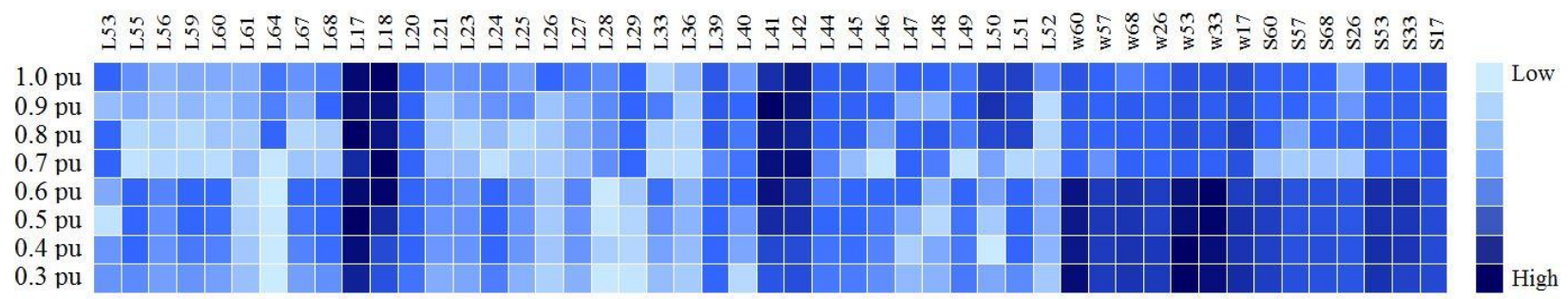

Fig. 7. Heatmap of ranking for small-disturbance stability index showing different system loading.

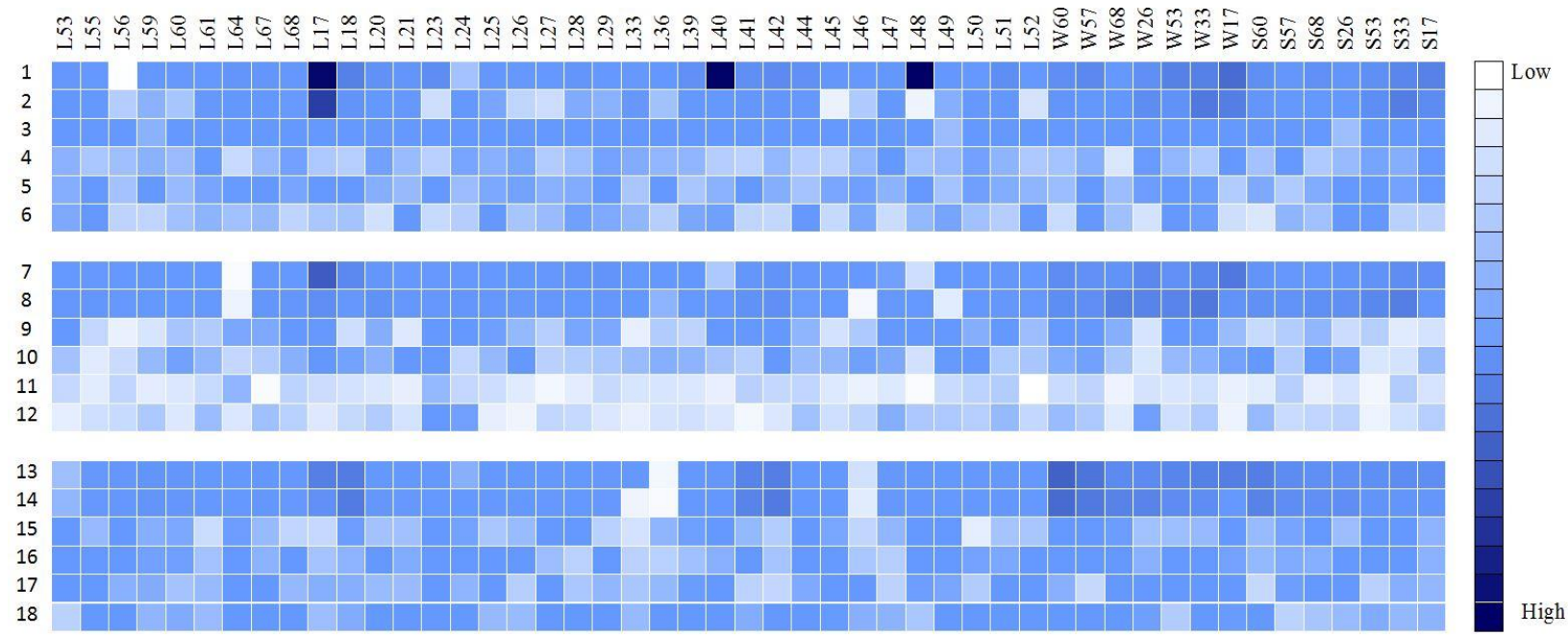

Fig. 8. Heatmap of ranking for transient stability index showing different system loading. 


\section{ILLUSTRATIVE RESULTS AND DISCUSSION}

\section{A. Priority Ranking for Voltage Stability}

Power system voltage stability analysis is performed by running PV-curve analysis. During the analysis, the loading level of the test network is varied to account for daily load variation, i.e., system load following the daily loading curve [48]. For a particular system loading level, e.g., $0.5 \mathrm{pu}$, the base starting point for load and generation is $0.5 \mathrm{pu}$. The subsequent $\mathrm{P}-\mathrm{V}$ curves are generated by increasing the system loading from this level. All loads are scaled to increase simultaneously through an iteration control with adaptive step size. The conventional generation is correspondingly scaled up to compensate the increment in load. The initial step size is set to be $0.5 \%$, the maximum step size is $2 \%$ and the minimum step size is $0.01 \%$. The step size reduces as the system approaches the stability limit. The iterations continue until the point when the load flow calculation doesn't converge anymore and this point is taken as voltage stability limit (system loadability limit). The active power $\mathrm{P}$ (MW) and the voltage magnitude $\mathrm{V}$ (pu) of the collapse points are recorded to obtain the nose-point locus for voltage stability as presented in Fig. 1. The same process is repeated for all eight considered system loading levels as presented in Table II.

Furthermore, the impact of system uncertainties (actual load, wind speed and solar irradiation) on the location of P-V curve nose-point has been assessed through sensitivity analysis approach, Morris screening method. Thus, Fig. 6 shows a heatmap for the ranking of critical parameters affecting voltage stability through MSSA (Morris screening sensitivity analysis) under eight loading conditions selected from the daily loading curve. Table III lists top 5 critical parameters since they have the dominant influence on the change of loadability compared to the rest of the parameters. As the largest load in the system, L17 remains the most influential parameter at all loading levels. The penetration levels of the RES increase in the system according to equations (14) and (15) as $P_{L}$ and $G_{S M}$ decrease while $G_{R E S}$ is kept constant. Hence the uncertainties of RES become more influential and get higher ranks in high $P L_{R E S}$ cases compared to low $P L_{R E S}$ cases.

As can be seen from Table III, in the top 5 ranked critical parameters, the influence of uncertainties in loads 18, 42 and 41 on system voltage stability become less important with higher RES penetration level. With lower RES penetration level, the critical parameters are mostly the large loads in the system, such as $L_{17}, L_{18}, L_{42}, L_{41}$ and $L_{20}(6000 \mathrm{MW}, 2470 \mathrm{MW}$, $1000 \mathrm{MW}, 1150 \mathrm{MW}$ and $680 \mathrm{MW}$, respectively). Table III also reveals that the influence of RES generation on system voltage stability becomes important (such as W17, S17, W33, W68, W53 in the three right most columns) with lower loading (and at higher penetration of RES).

\section{B. Priority Ranking for Small-disturbance Stability}

Fig. 7 shows the heatmap for the ranking of critical parameters affecting small-disturbance stability through MSSA under 8 loading conditions selected from the daily loading curve. Table IV presents the top 5 critical parameters. It can be seen from Fig. 7 that the importance of uncertainties of RES generation increases as $P L_{R E S}$ increases. Loads 17 and 18 are found to have a dominant impact on system small-disturbance stability at all loading levels. The uncertainty of loads 41 and 42 become influential only under high loading scenarios where
TABLE III

TOP 5 INFLUENTIAL PARAMETERS FOR VOLTAGE STABILITY FOLLOWING DAILY LOADING CURVE

\begin{tabular}{|c|c|c|c|c|c|c|c|c|}
\hline & \multicolumn{8}{|c|}{ Variable Loading Conditions Selected from Daily Loading Curve } \\
\hline & $1.0 \mathrm{pu}$ & $0.9 \mathrm{pu}$ & $0.8 \mathrm{pu}$ & $0.7 \mathrm{pu}$ & $0.6 \mathrm{pu}$ & $0.5 \mathrm{pu}$ & $0.4 \mathrm{pu}$ & $0.3 \mathrm{pu}$ \\
\hline \multirow{5}{*}{ 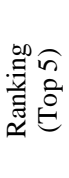 } & L17 & L17 & L17 & $\mathrm{L} 17$ & L17 & L17 & L17 & L17 \\
\hline & L18 & W17 & W17 & W17 & W17 & W17 & W17 & W17 \\
\hline & L42 & L18 & L18 & W53 & S17 & S17 & S17 & W33 \\
\hline & L41 & S17 & S17 & S17 & W26 & W68 & W68 & S17 \\
\hline & L20 & L20 & L20 & L18 & L18 & W53 & W33 & W53 \\
\hline
\end{tabular}

TABLE IV

TOP 5 INFLUENTIAL PARAMETERS FOR SMALL-DISTURBANCE STABILITY FOLLOWING DAILY LOADING CURVE

Variable Loading Conditions Selected from Daily Loading Curve

\begin{tabular}{|c|c|c|c|c|c|c|c|c|}
\hline & $1.0 \mathrm{pu}$ & $0.9 \mathrm{pu}$ & $0.8 \mathrm{pu}$ & $0.7 \mathrm{pu}$ & $0.6 \mathrm{pu}$ & $0.5 \mathrm{pu}$ & $0.4 \mathrm{pu}$ & $0.3 \mathrm{pu}$ \\
\hline \multirow{5}{*}{ 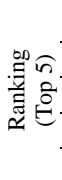 } & L18 & L41 & L17 & L18 & W33 & L17 & W53 & W53 \\
\hline & L17 & L17 & L18 & $\mathrm{L} 42$ & L18 & W33 & W33 & W60 \\
\hline & $\mathrm{L} 42$ & L18 & L41 & L41 & L17 & W53 & L17 & W33 \\
\hline & L41 & $\mathrm{L} 42$ & $\mathrm{~L} 42$ & L17 & L41 & W60 & W60 & L17 \\
\hline & L51 & L50 & W17 & W17 & W53 & L18 & S53 & W17 \\
\hline
\end{tabular}

L=Bus Loading, W=Wind Farm, S=PV Farm, Numbering corresponding to system buses in Figure 5

$P L_{R E S}$ is lower than $30 \%$, however their influence is diminishing as $P L_{R E S}$ increases and the influence (ranking) of wind farms 60, 53, and 33 becomes higher. The loads 50 and 51 is also ranked high at higher loading levels, however, their importance diminishes when the system loading reduces below 0.9 p.u.

\section{Priority Ranking for Transient Stability}

The transient stability of the system is analyzed by calculating the rotor angle displacement between generators after a large disturbance in the system. The disturbance considered was a three-phase fault on the line followed by the line disconnection. The fault duration is considered as 13 cycles [49]. (Note that longer than usual fault durations of 13 cycles are adopted to ensure sufficient number of unstable cases with considered test network. Otherwise one would typically consider faults durations of 4-7 cycles at transmission system level $[29,49])$ In this study several different fault locations were used. Three loading levels (1.0pu, $0.6 \mathrm{pu}$ and $0.3 \mathrm{pu}$ ) are selected from the daily loading curve for demonstration purposes.

The faults were applied on six different lines, namely line 12 (between buses 21 and 68, near critical generator G9), line 56 (between buses 33 and 38, near critical generator G11), line 42 (between buses 60 and 61, tie-line between NETS-NYPS), line 45 (between buses 53 and 54, tie-line between NETS-NYPS), line 70 (between buses 40 and 41, tie-line between NYPS-G14) and line 72 (between buses 18 and 50, tie-line between NYPS-G16).

In Fig. 8, rows 1-6, 7-12, and 13-18 show the importance ranking of system parameters for $1.0 \mathrm{pu}, 0.6 \mathrm{pu}$, and $0.3 \mathrm{pu}$ system loading, respectively. Each group of six rows as mentioned above represent the results for faults at lines 12, 56, 42, 45, 70 and 72, respectively. For faults on lines 12 and 56, the uncertainties in large system loads (L17, L18, L40 etc.) are identified as critical. These lines are closer to critical generators G9 and G11, which are identified to be the most unstable generators in NYPS and NETS area due to the fact that they have relatively smaller moment of inertia constant. However as 


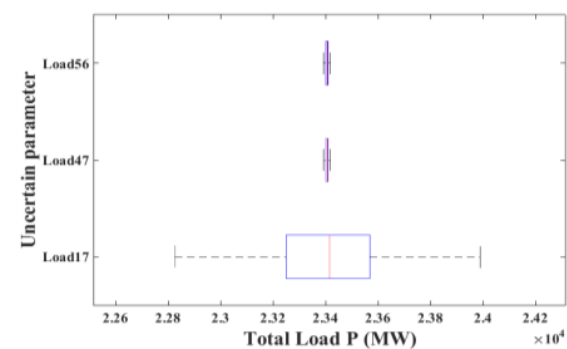

Fig. 9. Variation of voltage stability index when individual parameter is modelled as uncertain (keeping all other constant).

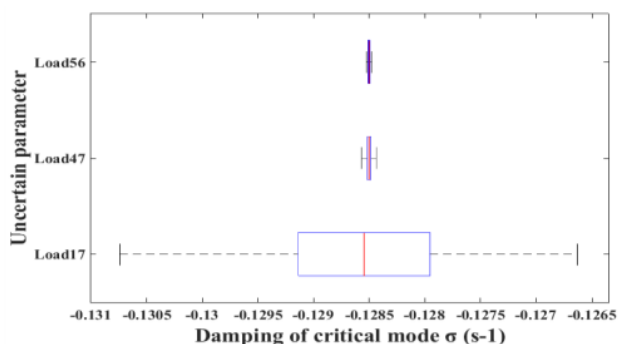

Fig. 10. Variation of small-disturbance stability index when individual parameter is modelled as uncertain (keeping all other constant).

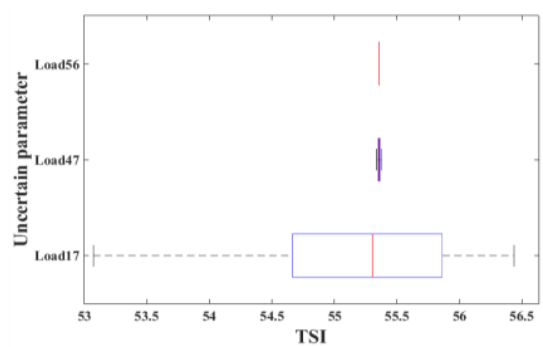

Fig. 11. Variation of transient stability index when individual parameter is modelled as uncertain (keeping all other constant).

TABLE V

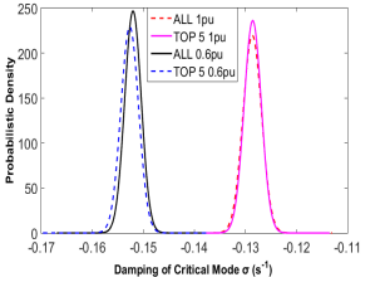

Fig. 13. $p d f$ s for Case Studies 5 to 8

Fig. 15. Scatter Plots for Case Studies 5 to 8

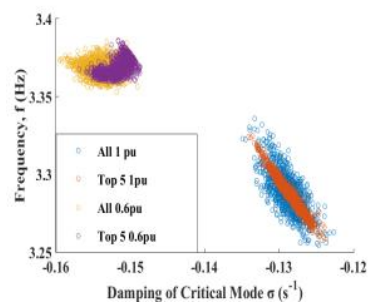

Fig. 14. Scatter plots for Case Studies 1 to 4 the proportion of RES penetration level increases, the importance of RES increases, and hence wind and PV generators are coming on the top of the priority list (as shown in Fig. 8)

\section{Validation of Ranking Results obtained through MSSA}

Previous studies in [19] and [17] indicated that MSSA delivers a good ranking of critical parameters compared to commonly used GSAs. This paper validates the ranking results obtained through MSSA by plotting $p d f \mathrm{~s}$ of the sensitivity indices for different stability analysis when all the uncertainties are modelled probabilistically and when only the top 5 selected uncertainties are modelled. Several case studies $\left(C S_{1}-C S_{8}\right)$, as listed in Table $\mathrm{V}$, are considered here to examine parameter ranking for voltage and small-disturbance stability. The boxplots are used to illustrate the influence of selected (based on their ranking) uncertain parameters on system stability. The parameters considered are load 17 (L17), identified as the most critical parameter for voltage and angular stability, and load 47 (L47) and load 56 (L56) identified as parameters with moderate and low influence on system voltage and angular stability, respectively.

Figs. 9, 10 and 11 illustrate the system voltage and angular stability behaviour affected by selected uncertain parameters. It can be seen from Fig. 9 that the variation of system loadability is affected significantly when L17 (i.e the most critical load) is modelled as uncertain parameters keeping all other constant at their base value. On the other hand, when L47 or L56 are modelled individually as uncertain parameters (keeping all
PROPOSED CASE STUDIES FOR THE VALIDATION OF RANKING RESULTS

\begin{tabular}{|c|c|c|c|c|c|c|c|}
\hline \multirow{5}{*}{ 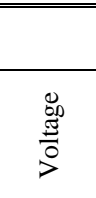 } & & \multicolumn{5}{|c|}{ Modelled Uncertainties } & $\begin{array}{l}\text { Loading } \\
\text { Condition }\end{array}$ \\
\hline & $C S_{1}$ & & & ALL & & & \multirow{2}{*}{$1.0 \mathrm{pu}$} \\
\hline & $C S_{2}$ & L17 & L18 & $\mathrm{L} 42$ & L41 & L20 & \\
\hline & $C S_{3}$ & \multicolumn{5}{|c|}{ ALL } & \multirow{2}{*}{$0.6 \mathrm{pu}$} \\
\hline & $C S_{4}$ & L17 & W17 & S17 & W26 & L18 & \\
\hline \multirow{4}{*}{ 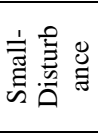 } & $C S_{5}$ & & & ALL & & & \multirow{2}{*}{$1.0 \mathrm{pu}$} \\
\hline & $C S_{6}$ & L18 & L17 & $\mathrm{L} 42$ & L41 & L51 & \\
\hline & $C S_{7}$ & & & ALL & & & \multirow{2}{*}{$0.6 \mathrm{pu}$} \\
\hline & $C S_{8}$ & W33 & L18 & L17 & L41 & W53 & \\
\hline
\end{tabular}

others constant) then the variations in system loadability are insignificant compared to the previous case. The same trend is also observed for small-disturbance and transient stability, as shown in Fig. 10 and 11, respectively. It reveals the fact that identifying the most critical parameter is very important for system operation and by doing this the resource and effort can be dedicated for accurate modelling of a small number of important parameters only.

The pdfs of the probabilistic load margin and the probabilistic damping of the critical mode are plotted in Fig. 12 and Fig. 13 for different loading levels for the purpose of validation of the ranking results for voltage and small-disturbance stability, respectively. Fig. 14 and 15 plot the scatter plots for the system dynamic behaviour for case studies 1 to 8 . It can be seen from Fig. 12 that the uncertainties of the identified five most important parameters have the same (overlapping $p d f s$ for two different loading levels) impact on system voltage stability as combined impact of all uncertain parameters in the system. The same trend can be also observed in the scatter plot of Fig. 14 where the area of system nose-point dispersion for the five influential parameters covers a larger portion of the range of system load margin considering all 49 parameters, hence, the criticality of the system stability (measured by the system load margin) has been properly captured by the identified five most influential parameters. Similar conclusions related to the effect of the five most important parameters on small disturbance stability (damping of the critical eigenvalue) can be drown from $p d f s$ shown in Fig. 13 and scatter plot of critical eigenvalues shown in Fig 15. The results shown in Figs. 12-15 therefore, confirm that the system dynamic behaviour can be controlled to a large extent by tuning/reducing uncertainty of the set of critical parameters only as the rest of the uncertain parameters have significantly lower effect.

Fig. 13 and 15 shows the results of comparison of the effect of modelling selected number of uncertain parameters for small-disturbance stability. Similarly as in Fig. 12 and 14, it demonstrates that a subset of the most influential system 
parameters ( 5 in this case) have the dominant impact on power system small-disturbance stability and by carefully monitoring and controlling of these parameters the overall system small-disturbance stability can be improved.

\section{E. Ranking considering the Correlations between Uncertainties}

This section of the paper explores the influence of the correlations between modelled uncertainties on system stability. System uncertainties and their correlation patterns have been obtained from [50, 51]. Fig. 16 shows a $49 \times 49$ matrix of Pearson correlation coefficients between 49 system uncertain parameters. In the figure, row/column 1-35 represent system loads, 36-42 represent wind speed and 43-49 represent solar irradiance.

Fig. 16 clearly illustrates the intra-dependence and inter-dependence within parameter groups. For example in Fig. 16, five groups of closely located loads can be identified through their high intra-dependence structure. These load-load intra-dependence structures are influenced by the different lifestyles of consumers which depend on factors such as weather condition. The intra-dependence structures of wind-wind and PV-PV correlations are mainly determined by the distance between generation plants. This means closely located RES generation can be highly correlated as the weather pattern tends to be similar. However when the sampling time scale is relatively long and covers the whole year, the PV-PV correlation increases due to the fact that PV generation is now mainly dependent on day-time hours. The inter-dependence between load-wind and wind-PV is low, and this indicates load-wind and wind-PV are very poorly correlated. The inter-dependence between load-PV is relatively high, this is true considering that the day-time hours will change depending on seasons and people may turn on the heater/AC during the corresponding season.

As previous study [26] indicated that multivariate Gaussian copula is an efficient and accurate method for stochastic dependence structure modelling across all levels of RES penetration, the multivariate Gaussian copula was used here as well. Fig. 17 shows a heatmap and indicates the identified critical parameters when correlation between system parameters is considered.

The first row of the heatmap in Fig. 17 illustrates the ranking of the influence of system input parameters on voltage stability through the measure of system loadability. The top ranking parameters appear as groups instead of as single parameters (which was the case when no correlation was considered among the input parameters). The critical parameters are identified as loads on buses 17, 18, 20, 21, 23, 24, 25 and 68.

The second row of the heatmap in Fig. 17 shows the ranking of the critical parameters for small-disturbance stability through the measure of damping of critical eigenvalues. Once more the top ranking parameters appear as groups compared to the non-correlated case in Section V, $B$. The critical parameters are identified as load on buses 17 25 and 41 49.

The rows 3-8 of the heatmap in Fig. 17 reveal the ranking of critical parameters for transient stability through the measure of TSI. Rows 3-8 show the rankings for faults on lines $12,56,42$, 45, 70 and 72, respectively. The group of system loads from L41 to L49 is identified as a critical parameter affecting transient stability performance.

Overall Fig. 17 demonstrates that the groups of system load from L17 to L25 and L41 to L49 have the dominant influence over system voltage and angular stability. The accurate modelling of the correlation between uncertainties should be performed with extra care to ensure the stable operation of the power system.

By comparing the ranking of identified critical parameters between Sections $E$ and Sections $A, B$ and $C$ of Section V, the importance of correlation modelling of system input parameters can be deduced. When independent probabilistic modelling of system parameters is applied, the loss of correlation between the dataset may lead to ignoring an important parameter which may not be influential on its own but is highly correlated to an influential parameter. As shown in Fig. 16, high correlation can appear between load-load, load-PV, PV-PV and wind-wind. This characteristic of realistic system parameters makes the correlation modelling of system parameters very important for stability analysis.

\section{CONCLUSIONS}

This paper employs sensitivity analysis for the ranking of

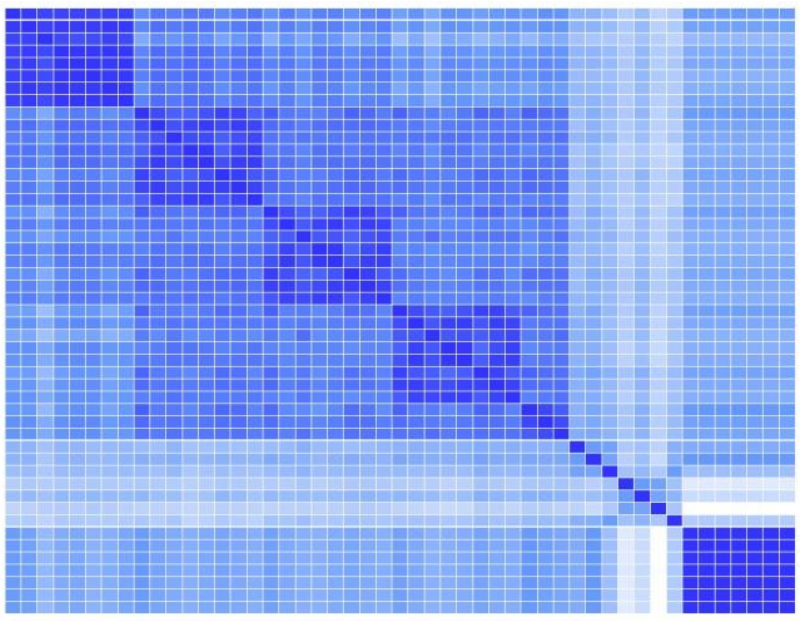

Fig. 16. Stochastic dependence structure of NETS-NYPS load, wind speed and solar irradiance over a year.

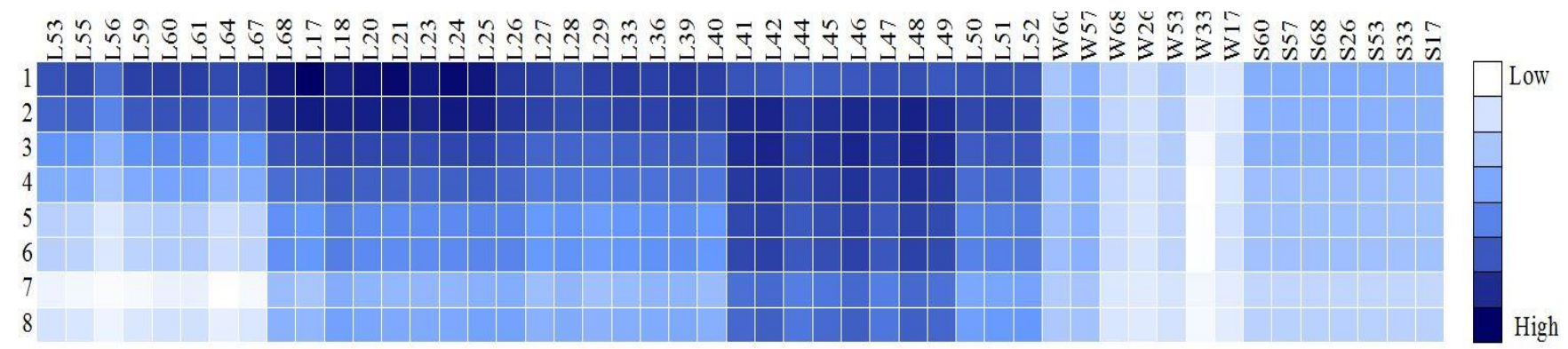

Fig. 17. Ranking of critical parameters for voltage and angular stability when correlations between input parameters are considered. 
critical parameters affecting voltage and angular stability in a system with RES generation. It represents the first study to include the identification of influential parameters affecting different stability problems in order to unveil the group of parameters which are considered critical to system voltage and angular stability as a whole. Independent probabilistic modelling of uncertainties in system load and RES generation is applied to a system input dataset. The sensitivity indices chosen for voltage, small-disturbance and transient stability are load margin, damping of the critical eigenvalues and TSI, respectively. The Morris screening method is used for the ranking of critical parameters followed by application of multivariate Gaussian copula for the correlation modelling of input parameters (load, wind speed and solar irradiation).

The large system loads are found to be critical for all types of stability when the RES penetration level is lower than $30 \%$. When the RES penetration level becomes higher than $30 \%$, the influence of uncertainties in RES generation increases and gets higher as the system inertia decreases due to conventional generator disconnection.

Analysis of real data from the actual network illustrates that a high level of intra-dependence and inter-dependence exist between system input parameters. Hence, the accurate modelling of correlation between parameters has to be considered when performing stability analysis. The results obtained in this paper reveal the disadvantage of independent modelling of system parameters as the critical parameters appear as groups after the input dataset is correlated. Hence, even though some of the system parameters may be uninfluential on their own, their variation/uncertainty can have a significant impact on system dynamic behaviour due to their correlation with other influential parameters.

The identification of critical parameters influencing system stability helps to narrow down the number of parameters in the system that need to be modelled accurately and reduces both personnel and financial resources required. The variation of system stability margin due to system parameter uncertainties can be converted to the critical parameter uncertainty margin and controlled by the detailed modelling of the important parameters only.

Building on the approach proposed in this paper the system behaviour under various operating conditions can be mapped into the system security operation profile and greatly facilitate stable and secure operation of the system.

\section{REFERENCES}

[1] R. Preece, N. C. Woolley, and J. V. Milanović, "The probabilistic collocation method for power-system damping and voltage collapse studies in the presence of uncertainties," IEEE Transactions on Power Systems, vol. 28, pp. 2253-2262, 2013.

[2] C. W. Group, "Review of the current status of tools and techniques for risk-based and probabilistic planning in power systems," $W G$ 601 of SC C4, 2010.

[3] W. Li and J. Zhou, "Probabilistic reliability assessment of power system operations," Electric Power Components and Systems, vol. 36, pp. 1102-1114, 2008.

[4] J. McCalley, S. Asgarpoor, L. Bertling, R. Billinion, H. Chao, J. Chen, et al., "Probabilistic security assessment for power system operations," in Power Engineering Society General Meeting, 2004. IEEE, 2004, pp. 212-220.

[5] S. Bu, W. Du, H. Wang, Z. Chen, L. Xiao, and H. Li, "Probabilistic analysis of small-signal stability of large-scale power systems as affected by penetration of wind generation," IEEE Transactions on Power Systems, vol. 27, pp. 762-770, 2012.

[6] H. Huang, C. Chung, K. W. Chan, and H. Chen, "Quasi-Monte Carlo based probabilistic small signal stability analysis for power systems with plug-in electric vehicle and wind power integration," IEEE Transactions on Power Systems, vol. 28, pp. 3335-3343, 2013.

[7] B. Qi, Y. Zhu, and J. Milanovic, "Probabilistic ranking of critical parameters affecting voltage stability in network with renewable generation," in PES Innovative Smart Grid Technologies Conference Europe (ISGT-Europe), 2016 IEEE, 2016, pp. 1-6.

[8] Y. Zhu, B. Qi, and J. V. Milanovic, "Probabilistic ranking of power system loads for voltage stability studies in networks with renewable generation," in PES Innovative Smart Grid Technologies Conference Europe (ISGT-Europe), 2016 IEEE, 2016, pp. 1-6.

[9] W. Wu, K. Wang, G. Li, and Y. Hu, "A stochastic model for power system transient stability with wind power," in PES General Meeting $\mid$ Conference \& Exposition, 2014 IEEE, 2014, pp. 1-5.

[10] L. Shi, S. Sun, L. Yao, Y. Ni, and M. Bazargan, "Effects of wind generation intermittency and volatility on power system transient stability," IET Renewable Power Generation, vol. 8, pp. 509-521, 2013.

[11] A. Adrees, J. Song, and J. V. Milanović, "The influence of different storage technologies on large power system frequency response," in Power Electronics and Motion Control Conference (IPEMC-ECCE Asia), 2016 IEEE 8th International, 2016, pp. 257-263.

[12] A. Adrees and J. V. Milanovic, "Study of frequency response in power system with renewable generation and energy storage," in Power Systems Computation Conference (PSCC), 2016, 2016, pp. 1-7.

[13] E. Vaahedi, W. Li, T. Chia, and H. Dommel, "Large scale probabilistic transient stability assessment using BC Hydro's on-line tool," IEEE Transactions on Power Systems, vol. 15, pp. 661-667, 2000.

[14] J. L. Rueda, D. G. Colomé, and I. Erlich, "Assessment and enhancement of small signal stability considering uncertainties," IEEE Transactions on power systems, vol. 24, pp. 198-207, 2009.

[15] J. Zhang, C. Tse, K. Wang, and C. Chung, "Voltage stability analysis considering the uncertainties of dynamic load parameters," IET generation, transmission \& distribution, vol. 3, pp. 941-948, 2009.

[16] J. Rueda and D. Colome, "Probabilistic performance indexes for small signal stability enhancement in weak wind-hydro-thermal power systems," IET generation, transmission \& distribution, vol. 3, pp. 733-747, 2009.

[17] B. Qi and J. V. Milanovic, "Identification of critical parameters affecting voltage stability in networks with renewable generations using sensitivity analysis methods," in PowerTech, 2017 IEEE Manchester, 2017, pp. 1-6.

[18] Y. Zhu and J. V. Milanović, "Efficient identification of critical load model parameters affecting power system voltage stability," in PowerTech, 2017 IEEE Manchester, 2017, pp. 1-6.

[19] K. N. Hasan, R. Preece, and J. V. Milanović, "Priority ranking of critical uncertainties affecting small-disturbance stability using sensitivity analysis techniques," IEEE Transactions on Power Systems, vol. 32, pp. 2629-2639, 2017.

[20] H. Park, R. Baldick, and D. P. Morton, "A stochastic transmission planning model with dependent load and wind forecasts," IEEE Transactions on Power Systems, vol. 30, pp. 3003-3011, 2015.

[21] N. Zhang, C. Kang, C. Singh, and Q. Xia, "Copula based dependent discrete convolution for power system uncertainty analysis," IEEE Transactions on Power Systems, vol. 31, pp. 5204-5205, 2016.

[22] W. Wu, K. Wang, B. Han, G. Li, X. Jiang, and M. L. Crow, "A versatile probability model of photovoltaic generation using pair copula construction," IEEE Transactions on Sustainable Energy, vol. 6, pp. 1337-1345, 2015.

[23] P. Li, X. Guan, J. Wu, and X. Zhou, "Modeling dynamic spatial correlations of geographically distributed wind farms and constructing ellipsoidal uncertainty sets for optimization-based generation scheduling," IEEE Transactions on Sustainable Energy, vol. 6, pp. 1594-1605, 2015.

[24] H. V. Haghi and S. Lotfifard, "Spatiotemporal modeling of wind generation for optimal energy storage sizing," IEEE Transactions on Sustainable Energy, vol. 6, pp. 113-121, 2015. 
[25] M. T. Bina and D. Ahmadi, "Stochastic modeling for the next day domestic demand response applications," IEEE Transactions On Power Systems, vol. 30, pp. 2880-2893, 2015.

[26] K. N. Hasan and R. Preece, "Influence of Stochastic Dependence on Small-Disturbance Stability and Ranking Uncertainties," IEEE Transactions on Power Systems, 2017.

[27] R. Hentati and J.-L. Prigent, "Chapter 4 Copula Theory Applied to Hedge Funds Dependence Structure Determination," in Nonlinear Modeling of Economic and Financial Time-Series, ed: Emerald Group Publishing Limited, 2010, pp. 83-109.

[28] X. Xu, Z. Yan, M. Shahidehpour, H. Wang, and S. Chen, "Power System Voltage Stability Evaluation Considering Renewable Energy with Correlated Variabilities," IEEE Transactions on Power Systems, 2017.

[29] P. Kundur, Power system stability and control: McGraw-hill New York, 1994.

[30] P. N. Papadopoulos and J. V. Milanović, "Impact of penetration of non-synchronous generators on power system dynamics," in PowerTech, 2015 IEEE Eindhoven, 2015, pp. 1-6.

[31] P. N. Papadopoulos and J. V. Milanović, "Probabilistic framework for transient stability assessment of power systems with high penetration of renewable generation," IEEE Transactions on Power Systems, vol. 32, pp. 3078-3088, 2017.

[32] D. Gautam, V. Vittal, and T. Harbour, "Impact of increased penetration of DFIG-based wind turbine generators on transient and small signal stability of power systems," IEEE Transactions on power systems, vol. 24, pp. 1426-1434, 2009.

[33] J. M. Morales, L. Baringo, A. J. Conejo, and R. Mínguez, "Probabilistic power flow with correlated wind sources," IET generation, transmission \& distribution, vol. 4, pp. 641-651, 2010.

[34] G. Valverde, A. T. Saric, and V. Terzija, "Stochastic monitoring of distribution networks including correlated input variables," IEEE Transactions on Power Systems, vol. 28, pp. 246-255, 2013.

[35] H. Ahmadi and H. Ghasemi, "Maximum penetration level of wind generation considering power system security limits," IET Generation, Transmission \& Distribution, vol. 6, pp. 1164-1170, 2012.

[36] A. Soroudi, M. Aien, and M. Ehsan, "A probabilistic modeling of photo voltaic modules and wind power generation impact on distribution networks," IEEE Systems Journal, vol. 6, pp. 254-259, 2012.

[37] K. Zou, A. P. Agalgaonkar, K. M. Muttaqi, and S. Perera, "Distribution system planning with incorporating DG reactive capability and system uncertainties," IEEE Transactions on Sustainable Energy, vol. 3, pp. 112-123, 2012.

[38] A. Saltelli, K. Chan, and E. M. Scott, Sensitivity analysis vol. 1: Wiley New York, 2000.

[39] K. N. Hasan and R. Preece, "Impact of Stochastic Dependence within Load and Non-synchronous Generation on Frequency Stability," presented at the IREP'2017, Espinho, Portugal, 2017.

[40] M. Sklar, "Fonctions de repartition an dimensions et leurs marges," Publ. Inst. Statist. Univ. Paris, vol. 8, pp. 229-231, 1959.

[41] H. V. Haghi, M. T. Bina, and M. A. Golkar, "Nonlinear modeling of temporal wind power variations," IEEE Transactions on Sustainable Energy, vol. 4, pp. 838-848, 2013.

[42] MathWorks. (Accessed on 16th May 2018). "Simulating Dependent Random Variables Using Copulas". . Available: https://uk.mathworks.com/help/stats/examples/simulating-depende nt-random-variables-using-copulas.html\#responsive_offcanvas.

[43] R. Preece and J. V. Milanović, "Efficient estimation of the probability of small-disturbance instability of large uncertain power systems," IEEE Transactions on Power Systems, vol. 31, pp. 1063-1072, 2016.

[44] R. D. Zimmerman, C. E. Murillo-Sánchez, and R. J. Thomas, "MATPOWER: Steady-state operations, planning, and analysis tools for power systems research and education," IEEE Transactions on power systems, vol. 26, pp. 12-19, 2011.

[45] G. Rogers, Power system oscillations: Springer Science \& Business Media, 2012.

[46] G. Code, "General Generating Unit (and OTSDUW) Requirements," p. 19, July 2016.

[47] K. Yamashita, S. Djokic, J. Matevosyan, F. Resende, L. Korunovic, Z. Dong, et al., "Modelling and Aggregation of Loads in Flexible Power Networks-Scope and Status of the Work of CIGRE WG C4. 605," IFAC Proceedings Volumes, vol. 45, pp. 405-410, 2012.
"Future energy scenarios: UK gas and electricity transmission," National GridJuly 20152015.

T. Guo and J. V. Milanović, "Online identification of power system dynamic signature using PMU measurements and data mining," IEEE Transactions on Power Systems, vol. 31, pp. 1760-1768, 2016.

[50] A. ERCOT, TX, USA. (2017). Hourly Load Data Archives. Available: http://www.ercot.com/gridinfo/load/load_hist/

[51] AgriMet. (2017). Historical Dayfile Data Access. Available: https://www.usbr.gov/pn/agrimet/webaghrread.html

Buyang Qi (S'15) received a BEng degree in Electrical and Electronic Engineering from the University of Manchester, Manchester, U.K. in 2015. He is currently working towards a Ph.D. degree at the University of Manchester.

Kazi N. Hasan (GS'08-M'14) is working as a post-doctoral research associate with the University of Manchester, Manchester, U.K. His research interest includes analyzing the impact of distributed energy resources and new types of loads into the electricity grid.

Jovica V. Milanovi'c (M'95-SM'98-F'10) received the Dipl.Ing. and M.Sc. degrees from the University of Belgrade, Belgrade, Yugoslavia, the Ph.D. degree from the University of Newcastle, Newcastle, Australia, and the Higher Doctorate (D.Sc. degree) from the University of Manchester, Manchester, U.K., all in electrical engineering. Currently, he is a Professor of electrical power engineering, the Deputy Head of the School, and the Director of External Affairs in the School of Electrical and Electronic Engineering, University of Manchester, a Visiting Professor with the University of Novi Sad, Serbia, the University of Belgrade, Serbia, and a Conjoint Professor with the University of Newcastle, Australia. He is a Chartered Engineer in the U.K. He is a Foreign member of the Serbian Academy of Engineering Sciences, a Fellow of the IET, a Distinguished IEEE PES Lecturer, and currently serves on the IEEE PES Governing Board as a Regional Representative for Europe, Middle East, and Africa, and on the IEEE PES Fellows Committee. 